\begin{tabular}{|c|l|}
\hline Title & Sur la d'eg'en'erescence de quelques formules de connexion pour les fonctions hy perg'eom'etriques de Gauss \\
\hline Author(s) & Watanabe, Humihiko \\
\hline Citation & Hokkaido University Preprint Series in Mathematics, 613, 1-21 \\
\hline Issue Date & 2004-07-21 \\
\hline DOI & 10.14943/83758 \\
\hline Doc URL & http://hdl.handle.net/2115/69362 \\
\hline Type & bulletin (article) \\
\hline File Information & pre613.pdf \\
\hline
\end{tabular}

Instructions for use 


\title{
Sur la Dégénérescence de Quelques Formules de Connexion pour les Fonctions Hypergéométriques de Gauss
}

\author{
Humihiko Watanabe \\ Institut de Technologie de Kitami
}

Révisé au 20 juillet 2004;

Dédié à Professeur Tatsuo Suwa à l'occasion de son soixantième anniversaire.

\begin{abstract}
$\S 0$ Introduction
Il est bien connu qu'il existe une opération de limite qui fasse une dégénérescence de l'équation différentielle hypergéométrique de Gauss en l'équation différentielle hypergéométrique confluente. Evidemment, cette opération de limite ramène aussi les solutions de l'équation différentielle hypergéométrique à celles de l'équation différentielle hypergéométrique confluente; par example, elle ramène la série hypergéométrique de Gauss à la série hypergéométrique confluente de Kummer.

L'équation différentielle hypergéométrique admet vingt-quatre solutions locales appelées les solutions de Kummer ([4]), et il est connu qu'il existe, d'après Kummer [4] (voir aussi Goursat [2]), les relations linéaires à coefficients constants entre trois quelconques de ces solutions, qui sont appelées les formules de connexion. On peut considérer que ces relations linéaires définissent les prolongements analytiques des solutions de l'équation différentielle hypergéométrique. Donc, il nous semble important de faire un calcul explicite de la dégénérescence de ces relations linéaires par l'opération de limite mentionnée ci-dessus, car cette dégénérescence relie la théorie des prolongements analytiques des solutions de l'équation différentielle hypergéométrique à la théorie analogue sur l'équation différentielle hypergéométrique confluente. Le but de cet article est de calculer rigoureusement la dégénérescence de quelques formules de connexion. Bien que ce sujet soit très classique, nous ne trouvons aucun travail qui en traite clairement et rigoureusement.
\end{abstract}

Le plan est le suivant. Dans le paragraphe 1, nous faisons un calcul formel de la dégénérescence de la formule de connexion entre 0 et $\infty$, et soulevons quelques

165, Koencho, Kitami 0908507, Hokkaïdo, Japon; E-mail: hwatanab@cs.kitami-it.ac.jp 
questions se trouvant dans ce calcul formel. Dans le paragraphe 2, nous faisons un calcul rigoureux de la dégénérescence de la formule de connexion entre 0 et $\infty$. Dans le paragraphe 3, nous faisons un calcul analogue sur la formule de connexion entre 0 et 1 . Dans le paragraphe 4 , nous donnons la représantation intégrale de Pochhammer qui s'emploie dans les paragraphes 2 et 3 .

Remerciements. J'exprime toute ma gratitude à Dr. K. Iohara qui m'a donné quelques bons conseils pendant notre discussion à ce sujet. Je remercie aussi le personnel du Département de Mathématiques, l'Université de Kobé de m'avoir permis de consulter beaucoup de livres et de journaux mathématiques dans la bibliothèque.

\section{$\S 1$ Calcul formel}

Tout d'abord, nous introduisons la définition suivante:

Définition. (Une navigation circulaire sur le Lac Saroma du Hokkaïdo) Soit a un nombre réel, et soit $\lambda$ un nombre complexe. Supposons que la fonction linéaire complexe $a-z$ de la variable $z$ prend l'argument 0 sur la demi-droite réelle $z<a$ : $\arg (a-z)=0$. Alors, on a $\arg (z-a)=\pi$ ou bien $\arg (z-a)=-\pi$ sur cette demi-droite. Au cas où $\arg (z-a)=\pi$, puisqu'on a $(a-z)^{\lambda}=e^{-\pi i \lambda}(z-a)^{\lambda}$, nous écrirons $(a-z)^{\lambda}=\left(a+e^{-\pi i} z\right)^{\lambda}$. Au contraire, au cas où $\arg (z-a)=-\pi$, puisqu'on a $(a-z)^{\lambda}=e^{\pi i \lambda}(z-a)^{\lambda}$, nous écrirons $(a-z)^{\lambda}=\left(a+e^{\pi i} z\right)^{\lambda}$.

Dans ce qui suit, nous utiliserons fréquemment la notation de cette définition pour choisir précisément une détermination d'une fonction multiforme.

Considérons l'équation différentielle hypergéométrique de Gauss:

$$
z(1-z) \frac{d^{2} w}{d z^{2}}+\{\gamma-(\alpha+\beta+1) z\} \frac{d w}{d z}-\alpha \beta w=0
$$

où $z$ désigne la variable indépendante, $w$ la fonction inconnue, et où $\alpha, \beta, \gamma$ désignent des constantes complexes. On voit aisément que la nouvelle équation différentielle obtenue par le remplacement de la variable $z$ par $z / \beta$ dans (1.1) se ramène à l'équation différentielle hypergéométrique confluente

$$
z \frac{d^{2} w}{d z^{2}}+(\gamma-z) \frac{d w}{d z}-\alpha w=0
$$

lorsque $\beta$ tend vers l'infini $\infty$. Nous voudrions prendre la même limite $\beta \rightarrow \infty$ pour la formule obtenue par le remplacement de la variable $z$ par $z / \beta$ dans la formule 
de connexion de Gauss:

$$
\begin{aligned}
F(\alpha, \beta, \gamma, z)= & \frac{\Gamma(\gamma) \Gamma(\beta-\alpha)}{\Gamma(\beta) \Gamma(\gamma-\alpha)}\left(e^{-\pi i} z\right)^{-\alpha} F\left(\alpha, 1+\alpha-\gamma, 1+\alpha-\beta, z^{-1}\right) \\
& +\frac{\Gamma(\gamma) \Gamma(\alpha-\beta)}{\Gamma(\alpha) \Gamma(\gamma-\beta)}\left(e^{-\pi i} z\right)^{-\beta} F\left(\beta, 1+\beta-\gamma, 1+\beta-\alpha, z^{-1}\right)
\end{aligned}
$$

où $F(\alpha, \beta, \gamma, z)$ désigne la série hypergéométrique de Gauss ayant le domaine de convergence $|z|<1$, et où $\Gamma(t)$ désigne la fonction Gamma. Remarquons que la formule (1.3) est valable sous les conditions: $\gamma \neq 0,-1,-2, \ldots$, et $\alpha-\beta \notin \mathbf{Z}$. Les deux fonctions $\left(e^{-\pi i} z\right)^{-\alpha} F\left(\alpha, 1+\alpha-\gamma, 1+\alpha-\beta, z^{-1}\right)$ et $\left(e^{-\pi i} z\right)^{-\beta} F\left(\beta, 1+\beta-\gamma, 1+\beta-\alpha, z^{-1}\right)$ dans le deuxième membre de (1.3) sont considérées comme fonctions uniformes sur le domaine $D=\{z \in \mathbf{C}|| z \mid>1\}-\{z \in$ $\mathbf{R} \mid z \geq 0\}$ telles que leur variable $z$ prenne l'argument $\pi$ sur la demi-droite réelle $z<-1$; elles forment un système fondamental des solutions de l'équation (1.1) en chaque point de $D$, pourvu que les constantes $\alpha$ et $\beta$ vérifient la condition $\alpha-\beta \notin \mathbf{Z}$. Ainsi, on peut considérer la formule (1.3) comme relation entre les trois solutions de (1.1), relation obtenue par le prolongement analytique de ce système fondamental sur le domaine $\{z \in \mathbf{C}|| z \mid<1\}-\{z \in \mathbf{R} \mid z \geq 0\}$. Prenons la limite formelle $\beta \rightarrow \infty$ pour la formule obtenue par le remplacement de $z$ par $z / \beta$ dans (1.3):

$$
\begin{aligned}
F\left(\alpha, \beta, \gamma, \frac{z}{\beta}\right)= & \frac{\Gamma(\gamma) \Gamma(\beta-\alpha)}{\Gamma(\beta) \Gamma(\gamma-\alpha)}\left(e^{-\pi i} \frac{z}{\beta}\right)^{-\alpha} F\left(\alpha, 1+\alpha-\gamma, 1+\alpha-\beta, \frac{\beta}{z}\right) \\
& +\frac{\Gamma(\gamma) \Gamma(\alpha-\beta)}{\Gamma(\alpha) \Gamma(\gamma-\beta)}\left(e^{-\pi i} \frac{z}{\beta}\right)^{-\beta} F\left(\beta, 1+\beta-\gamma, 1+\beta-\alpha, \frac{\beta}{z}\right)
\end{aligned}
$$

Mais, on voit aussitôt que le deuxième terme dans le deuxième membre de (1.4) diverge lorsque $\beta$ tend vers $\infty: \lim _{\beta \rightarrow \infty}\left(e^{-\pi i} z / \beta\right)^{-\beta} F(\beta, 1+\beta-\gamma, 1+\beta-\alpha, \beta / z)=$ $\infty$. Donc, il est impossible de prendre directement la limite $\beta \rightarrow \infty$ pour la formule (1.4). Pour éviter cette difficulté, nous remplaçons dans (1.3) la fonction $\left(e^{-\pi i} z\right)^{-\beta} F\left(\beta, 1+\beta-\gamma, 1+\beta-\alpha, z^{-1}\right)$ par la fonction $\left(e^{-\pi i} z\right)^{\alpha-\gamma}(1+$ $\left.e^{-\pi i} z\right)^{\gamma-\alpha-\beta} F\left(1-\alpha, \gamma-\alpha, \beta-\alpha+1, z^{-1}\right)$ d'après Kummer [4]:

$$
\begin{aligned}
F(\alpha, \beta, \gamma, z)= & \frac{\Gamma(\gamma) \Gamma(\beta-\alpha)}{\Gamma(\beta) \Gamma(\gamma-\alpha)}\left(e^{-\pi i} z\right)^{-\alpha} F\left(\alpha, 1+\alpha-\gamma, 1+\alpha-\beta, \frac{1}{z}\right) \\
& +\frac{\Gamma(\gamma) \Gamma(\alpha-\beta)}{\Gamma(\alpha) \Gamma(\gamma-\beta)}\left(e^{-\pi i} z\right)^{\alpha-\gamma}\left(1+e^{-\pi i} z\right)^{\gamma-\alpha-\beta} \\
& \times F\left(1-\alpha, \gamma-\alpha, \beta-\alpha+1, \frac{1}{z}\right)
\end{aligned}
$$


La fonction $\left(e^{-\pi i} z\right)^{\alpha-\gamma}\left(1+e^{-\pi i} z\right)^{\gamma-\alpha-\beta} F\left(1-\alpha, \gamma-\alpha, \beta-\alpha+1, z^{-1}\right)$ est considérée comme fonction uniforme sur $D$, et sa variable $z$ prend l'argument $\pi$ sur la demidroite réelle $z<-1$. En remplaçant $z$ par $z / \beta$ dans (1.5), on a

$$
\begin{aligned}
F\left(\alpha, \beta, \gamma, \frac{z}{\beta}\right)= & \frac{\Gamma(\gamma) \Gamma(\beta-\alpha)}{\Gamma(\beta) \Gamma(\gamma-\alpha)} \beta^{\alpha}\left(e^{-\pi i} z\right)^{-\alpha} F\left(\alpha, 1+\alpha-\gamma, 1+\alpha-\beta, \frac{\beta}{z}\right) \\
& +\frac{\Gamma(\gamma) \Gamma(\alpha-\beta)}{\Gamma(\alpha) \Gamma(\gamma-\beta)} \beta^{\gamma-\alpha}\left(e^{-\pi i} z\right)^{\alpha-\gamma}\left(1+e^{-\pi i} \frac{z}{\beta}\right)^{\gamma-\alpha-\beta} \\
& \times F\left(1-\alpha, \gamma-\alpha, \beta-\alpha+1, \frac{\beta}{z}\right) .
\end{aligned}
$$

Calculons la limite formelle $\beta \rightarrow \infty$ de chaque terme apparaissant dans (1.6). D'abord, on a facilement $\lim _{\beta \rightarrow \infty} F(\alpha, \beta, \gamma, z / \beta)=F(\alpha, \gamma, z)$, dont le deuxième membre est la série hypergéométrique confluente de Kummer ayant le domaine de convergence $|z|<\infty$. Ensuite, on trouve $\lim _{\beta \rightarrow \infty} \Gamma(\beta-\alpha) \beta^{\alpha} / \Gamma(\beta)=1$ d'après la formule de Stirling $\Gamma(t+1) \sim \sqrt{2 \pi t} t^{t} e^{-t}$ pour $t \rightarrow \infty$ dans le voisinage sectoriel $|\arg t| \square \pi-\delta$ avec un nombre réel positif quelconque $\delta$ ([1], [5]). Alors, s'il est permis de faire un calcul formel, on a

$$
\begin{gathered}
\lim _{\beta \rightarrow \infty} \frac{\Gamma(\gamma) \Gamma(\beta-\alpha)}{\Gamma(\beta) \Gamma(\gamma-\alpha)} \beta^{\alpha}\left(e^{-\pi i} z\right)^{-\alpha} F\left(\alpha, 1+\alpha-\gamma, 1+\alpha-\beta, \frac{\beta}{z}\right) \\
=\frac{\Gamma(\gamma)}{\Gamma(\gamma-\alpha)}\left(e^{-\pi i} z\right)^{-\alpha} \sum_{n=0}^{\infty} \frac{(-1)^{n}(\alpha)_{n}(\alpha-\gamma+1)_{n}}{n !} \frac{1}{z^{n}}
\end{gathered}
$$

où la série de puissance dans le deuxième membre est divergente et où $(\alpha)_{n}=$ $\alpha(\alpha+1) \cdots(\alpha+n-1)$. De même, par calcul formel, on a

$$
\begin{aligned}
\lim _{\beta \rightarrow \infty} & \frac{\Gamma(\gamma) \Gamma(\alpha-\beta)}{\Gamma(\alpha) \Gamma(\gamma-\beta)} \beta^{\gamma-\alpha}\left(e^{-\pi i} z\right)^{\alpha-\gamma}\left(1+e^{-\pi i} \frac{z}{\beta}\right)^{\gamma-\alpha-\beta} F\left(1-\alpha, \gamma-\alpha, \beta-\alpha+1, \frac{\beta}{z}\right) \\
& =\frac{\Gamma(\gamma)}{\Gamma(\alpha)} z^{\alpha-\gamma} e^{z} \sum_{n=0}^{\infty} \frac{(1-\alpha)_{n}(\gamma-\alpha)_{n}}{n !} \frac{1}{z^{n}}
\end{aligned}
$$

où la série de puissance dans le deuxième membre est aussi divergente. Enfin, en prenant la limite $\beta \rightarrow \infty$ pour (1.6), on obtient le développement asymptotique pour la fonction $F(\alpha, \gamma, z)$ à l'infini:

$$
\begin{aligned}
F(\alpha, \gamma, z) \sim & \frac{\Gamma(\gamma)}{\Gamma(\gamma-\alpha)}\left(e^{-\pi i} z\right)^{-\alpha} \sum_{n=0}^{\infty} \frac{(-1)^{n}(\alpha)_{n}(\alpha-\gamma+1)_{n}}{n !} \frac{1}{z^{n}} \\
& +\frac{\Gamma(\gamma)}{\Gamma(\alpha)} z^{\alpha-\gamma} e^{z} \sum_{n=0}^{\infty} \frac{(1-\alpha)_{n}(\gamma-\alpha)_{n}}{n !} \frac{1}{z^{n}} .
\end{aligned}
$$


Il y a encore deux problèmes dans le processus de ce calcul formel. L'un est de négliger encore la convergence dans quelques parts de ce calcul. L'autre est de ne pas avoir déterminé le voisinage sectoriel pour lequel est valable le développement asymptotique (1.7). La discussion dans les paragraphes suivants résoudra ces problèmes.

$\S 2$ Dégénérescence de la formule de connexion entre 0 et $\infty$

Nous commençons par la formule suivante:

$$
\begin{aligned}
& F\left(\alpha, \beta, \gamma, \frac{z}{\beta}\right)=\frac{\Gamma(\gamma) \Gamma(\beta-\alpha)}{\Gamma(\beta) \Gamma(\gamma-\alpha)} \beta^{\alpha}\left(e^{-\pi i} z\right)^{-\alpha} F\left(\alpha, 1+\alpha-\gamma, 1+\alpha-\beta, \frac{\beta}{z}\right)+ \\
& \quad+\frac{\Gamma(\gamma) \Gamma(\alpha-\beta)}{\Gamma(\alpha) \Gamma(\gamma-\beta)} \beta^{\gamma-\alpha}\left(e^{-\pi i} z\right)^{\alpha-\gamma}\left(1+e^{-\pi i} \frac{z}{\beta}\right)^{\gamma-\alpha-\beta} F\left(1-\alpha, \gamma-\alpha, \beta-\alpha+1, \frac{\beta}{z}\right)
\end{aligned}
$$

qui est valable pour trois nombres complexes $\alpha, \beta, \gamma$ tels que $\beta \neq 0, \gamma \neq 0,-1,-2, \ldots$, et $\alpha-\beta \notin \mathbf{Z}$. Soit $P(\alpha, \beta, \gamma, z)$ une détermination du premier terme du deuxième membre de (2.1), telle que, si $0<z<1$, $\arg z=0$ :

$$
P(\alpha, \beta, \gamma, z)=\frac{\Gamma(\gamma) \Gamma(\beta-\alpha)}{\Gamma(\beta) \Gamma(\gamma-\alpha)} \beta^{\alpha}\left(e^{-\pi i} z\right)^{-\alpha} F\left(\alpha, 1+\alpha-\gamma, 1+\alpha-\beta, \frac{\beta}{z}\right)
$$

Nous preuverons la

Proposition 2.1. Soient $\alpha, \gamma$ deux nombres complexes tels que

$$
\alpha \neq 1,2,3, \ldots, \quad \text { et } \gamma \neq 0,-1,-2, \ldots
$$

Soit $\theta$ un nombre réel tel que $\frac{1}{2} \pi<\theta<\pi$ ou $\pi<\theta<\frac{3}{2} \pi$. Alors, on a

$$
\begin{aligned}
\lim _{\beta \rightarrow e^{i \theta} \infty} P(\alpha, \beta, \gamma, z) & \\
& =\frac{\Gamma(\gamma) z^{-\alpha}}{\Gamma(\gamma-\alpha) \Gamma(\alpha)\left(e^{\pi i \alpha}-e^{-\pi i \alpha}\right)} \int_{e^{i(\theta-\pi)} \infty}^{(0+)} e^{-v} v^{\alpha-1}\left(1+\frac{v}{z}\right)^{\gamma-\alpha-1} d v
\end{aligned}
$$

et la convergence est localement uniforme sur le domaine $-\frac{3}{2} \pi<\arg z<\frac{3}{2} \pi$.

Démonstration. D'après le lemme 4.1, on a

$$
\begin{aligned}
P(\alpha, \beta, \gamma, z)= & \frac{\Gamma(\gamma) \Gamma(\beta-\alpha) \Gamma(1+\alpha-\beta) e^{\pi i \alpha} \beta^{\alpha} z^{-\alpha}}{\left(1-e^{2 \pi i \alpha}\right)\left(1-e^{-2 \pi i \beta}\right) \Gamma(\beta) \Gamma(\gamma-\alpha) \Gamma(\alpha) \Gamma(1-\beta)} \\
& \times \int^{(1+, 0+, 1-, 0-)} u^{\alpha-1}(1-u)^{-\beta}\left(1-\frac{\beta u}{z}\right)^{\gamma-\alpha-1} d u
\end{aligned}
$$


En substituant l'expression $u=e^{\pi i} \beta^{-1} v$ à l'intégrale définie du deuxième membre de l'égalité précédente, on a

$$
\begin{aligned}
& \int^{(1+, 0+, 1-, 0-)} u^{\alpha-1}(1-u)^{-\beta}\left(1-\frac{\beta u}{z}\right)^{\gamma-\alpha-1} d u \\
& =e^{\pi i \alpha} \beta^{-\alpha} \int^{\left(e^{-\pi i} \beta+, 0+, e^{-\pi i} \beta-, 0-\right)} v^{\alpha-1}\left(1+\frac{v}{\beta}\right)^{-\beta}\left(1+\frac{v}{z}\right)^{\gamma-\alpha-1} d v,
\end{aligned}
$$

d'où

$$
\begin{aligned}
P(\alpha, \beta, \gamma, z)= & \frac{\Gamma(\gamma) \Gamma(\beta-\alpha) \Gamma(1+\alpha-\beta) e^{2 \pi i \alpha} z^{-\alpha}}{\left(1-e^{2 \pi i \alpha}\right)\left(1-e^{-2 \pi i \beta}\right) \Gamma(\beta) \Gamma(\gamma-\alpha) \Gamma(\alpha) \Gamma(1-\beta)} \\
& \times \int^{\left(e^{-\pi i} \beta+, 0+, e^{-\pi i} \beta-, 0-\right)} v^{\alpha-1}\left(1+\frac{v}{\beta}\right)^{-\beta}\left(1+\frac{v}{z}\right)^{\gamma-\alpha-1} d v
\end{aligned}
$$

Posons $R=|\beta|$; considérons $\left(1-R^{-1}\right) e^{-\pi i} \beta$ comme point base du cycle de Pochhammer entourant $e^{-\pi i} \beta$ et 0 ; on peut écrire

$$
\begin{aligned}
& \int_{\left(1-R^{-1}\right) e^{-\pi i \beta} \beta}^{\left(e^{-\pi i} \beta+, 0+, e^{-\pi i} \beta-, 0-\right)} v^{\alpha-1}\left(1+\frac{v}{\beta}\right)^{-\beta}\left(1+\frac{v}{z}\right)^{\gamma-\alpha-1} d v \\
& =\int^{\left(e^{-\pi i} \beta+\right)}\left(1+\frac{v}{z}\right)^{\gamma-\alpha-1} d v \\
& +e^{-2 \pi i \beta} \int_{\left(1-R^{-1}\right) e^{-\pi i} \beta}^{(0+)} v^{\alpha-1}\left(1+\frac{v}{\beta}\right)^{-\beta}\left(1+\frac{v}{z}\right)^{\gamma-\alpha-1} d v \\
& +e^{2 \pi i(\alpha-\beta)} \int_{\left(1-R^{-1}\right) e^{-\pi i \beta}}^{\left(e^{-\pi i} \beta-\right)} v^{\alpha-1}\left(1+\frac{v}{\beta}\right)^{-\beta}\left(1+\frac{v}{z}\right)^{\gamma-\alpha-1} d v \\
& +e^{2 \pi i \alpha} \int_{\left(1-R^{-1}\right) e^{-\pi i} \beta}^{(0-)} v^{\alpha-1}\left(1+\frac{v}{\beta}\right)^{-\beta}\left(1+\frac{v}{z}\right)^{\gamma-\alpha-1} d v \\
& =\left(1-e^{2 \pi i \alpha}\right) \oint_{\left|v-e^{-\pi i} \beta\right|=1} v^{\alpha-1}\left(1+\frac{v}{\beta}\right)^{-\beta}\left(1+\frac{v}{z}\right)^{\gamma-\alpha-1} d v \\
& +\left(e^{-2 \pi i \beta}-1\right) \int_{\left(1-R^{-1}\right) e^{-\pi i} \beta}^{(0+)} v^{\alpha-1}\left(1+\frac{v}{\beta}\right)^{-\beta}\left(1+\frac{v}{z}\right)^{\gamma-\alpha-1} d v
\end{aligned}
$$

où $\oint_{\left|v-e^{-\pi i} \beta\right|=1}$ désigne l'intégrale qui est prise le long du cercle $\left|v-e^{-\pi i} \beta\right|=1$ dans le sens direct. En substituant le membre dernier de l'égalité précédente à l'intégrale définie de (2.5), on a

$$
\begin{aligned}
& P(\alpha, \beta, \gamma, z) \\
& =\frac{e^{2 \pi i \alpha} z^{-\alpha} \Gamma(\gamma) \Gamma(\beta-\alpha) \Gamma(1+\alpha-\beta)}{\left(1-e^{-2 \pi i \beta}\right) \Gamma(\alpha) \Gamma(\gamma-\alpha) \Gamma(\beta) \Gamma(1-\beta)} \oint_{\left|v-e^{-\pi i} \beta\right|=1} v^{\alpha-1}\left(1+\frac{v}{\beta}\right)^{-\beta}\left(1+\frac{v}{z}\right)^{\gamma-\alpha-1} d v \\
& +\frac{e^{2 \pi i \alpha} z^{-\alpha} \Gamma(\gamma) \Gamma(\beta-\alpha) \Gamma(1+\alpha-\beta)}{\left(e^{2 \pi i \alpha}-1\right) \Gamma(\alpha) \Gamma(\gamma-\alpha) \Gamma(\beta) \Gamma(1-\beta)} \int_{\left(1-R^{-1}\right) e^{-\pi i} \beta}^{(0+)} v^{\alpha-1}\left(1+\frac{v}{\beta}\right)^{-\beta}\left(1+\frac{v}{z}\right)^{\gamma-\alpha-1} d v
\end{aligned}
$$


dont le deuxième membre est valable sous la condition (2:3). Pour prendre la limite $\lim _{\beta \rightarrow e^{i \theta} \infty} P(\alpha, \beta, \gamma, z)$, il faut démontrer le

Lemme 2.2. Soit $\theta$ un nombre réel tel que $\frac{1}{2} \pi<\theta<\pi$ ou $\pi<\theta<\frac{3}{2} \pi$. On a:

(i) la quantité $\left|\frac{1}{1-e^{-2 \pi i \beta}}\right|$ est bornée pour $\beta \rightarrow e^{i \theta} \infty$;

(ii) $\lim _{\beta \rightarrow e^{i \theta} \infty} \frac{\Gamma(\beta-\alpha) \Gamma(1+\alpha-\beta)}{\Gamma(\beta) \Gamma(1-\beta)}=e^{-\pi i \alpha}$;

(iii) $\lim _{\beta \rightarrow e^{i \theta} \infty} \oint_{\left|v-e^{-\pi i} \beta\right|=1} v^{\alpha-1}\left(1+\frac{v}{\beta}\right)^{-\beta}\left(1+\frac{v}{z}\right)^{\gamma-\alpha-1} d v=0$.

Démonstration. (i) Evidente.

(ii) C'est le résultat immédiat des deux faits $\Gamma(\beta-\alpha) / \Gamma(\beta) \sim \beta^{\alpha}$ et $\Gamma(-\beta+1+\alpha) / \Gamma(-\beta+1) \sim\left(e^{\pi i} \beta\right)^{-\alpha}$ d'après [9].

(iii) On pose $\beta=\operatorname{Re}^{i \theta}(R>0)$; on donne la nouvelle variable $\varphi(\theta-2 \pi \square \varphi \square \theta)$ par la formule $v-e^{-\pi i} \beta=e^{i \varphi}$, d'où $d v=i e^{i \varphi} d \varphi$. Ainsi, on a

$$
\begin{aligned}
& \left|\oint_{\left|v-e^{-\pi i} \beta\right|=1} v^{\alpha-1}\left(1+\frac{v}{\beta}\right)^{-\beta}\left(1+\frac{v}{z}\right)^{\gamma-\alpha-1} d v\right| \\
= & \left|\int_{\theta-2 \pi}^{\theta}\left(R e^{i(\theta-\pi)}+e^{i \varphi}\right)^{\alpha-1}\left(\frac{e^{i(\varphi-\theta)}}{R}\right)^{-R e^{i \theta}}\left(1+\frac{R e^{i(\theta-\pi)}}{z}+\frac{e^{i \varphi}}{z}\right)^{\gamma-\alpha-1} i e^{i \varphi} d \varphi\right| \\
& \square \int_{\theta-2 \pi}^{\theta}\left|\left(e^{i(\theta-\pi)}+\frac{e^{i \varphi}}{R}\right)^{\alpha-1}\left(\frac{1}{R}+\frac{e^{i(\theta-\pi)}}{z}+\frac{e^{i \varphi}}{R z}\right)^{\gamma-\alpha-1} R^{\gamma-2}\right| e^{R[(\varphi-\theta) \sin \theta+\cos \theta \log R]} d \varphi .
\end{aligned}
$$

Ici, on a évidemment

$$
\lim _{R \rightarrow \infty}\left|\left(e^{i(\theta-\pi)}+\frac{e^{i \varphi}}{R}\right)^{\alpha-1}\left(\frac{1}{R}+\frac{e^{i(\theta-\pi)}}{z}+\frac{e^{i \varphi}}{R z}\right)^{\gamma-\alpha-1} R^{\gamma-2}\right| e^{R[(\varphi-\theta) \sin \theta+\cos \theta \log R]}=0
$$

car $\lim _{R \rightarrow \infty} \cos \theta \log R=-\infty$. Par conséquent, le membre dernier de l'inégalité précédente tend vers 0 lorsque $R$ tend vers $\infty$, ce qui preuve la formule (iii) de ce lemme.

D'après le lemme 2.2 , on aura aisément

$$
\begin{aligned}
\lim _{\beta \rightarrow e^{i \theta} \infty} & P(\alpha, \beta, \gamma, z) \\
& =\frac{\Gamma(\gamma) z^{-\alpha}}{\Gamma(\gamma-\alpha) \Gamma(\alpha)\left(e^{\pi i \alpha}-e^{-\pi i \alpha}\right)} \int_{e^{i(\theta-\pi)} \infty}^{(0+)} e^{-v} v^{\alpha-1}\left(1+\frac{v}{z}\right)^{\gamma-\alpha-1} d v
\end{aligned}
$$

où le deuxième membre, fonction de $z$, vérifie l'équation (1.2). Comme $\frac{1}{2} \pi<$ $\theta<\pi$ ou $\pi<\theta<\frac{3}{2} \pi$, l'intégrale définie dans le deuxième membre de (2.4) est 
certainement convergente (voir [3]). De plus, on voit que le deuxième membre de (2.4) est valable pour $z$ tel que $-\frac{3}{2} \pi<\arg z<\frac{3}{2} \pi, \operatorname{car} \theta-\pi<\arg \left(e^{\pi i} z\right)<\theta+\pi$, i.e., $\theta-2 \pi<\arg z<\theta$. On voit aisément que la convergence de (2.4) est localement uniforme sur le domaine $-\frac{3}{2} \pi<\arg z<\frac{3}{2} \pi$, si on regarde la démonstration du lemme 2.2. Ainsi la proposition 2.1 est démontrée.

Soit $Q(\alpha, \beta, \gamma, z)$ une détermination du deuxième terme du deuxième membre de (2.1), tell que, si $0<z<1, \arg z=0$ :

$$
\begin{aligned}
& Q(\alpha, \beta, \gamma, z) \\
& =\frac{\Gamma(\gamma) \Gamma(\alpha-\beta)}{\Gamma(\alpha) \Gamma(\gamma-\beta)} \beta^{\gamma-\alpha}\left(e^{-\pi i} z\right)^{\alpha-\gamma}\left(1+e^{-\pi i} \frac{z}{\beta}\right)^{\gamma-\alpha-\beta} F\left(1-\alpha, \gamma-\alpha, \beta-\alpha+1, \frac{\beta}{z}\right)
\end{aligned}
$$

Nous preuverons la

Proposition 2.3. Soient $\alpha, \gamma$ deux nombres complexes tels que

$$
\gamma \neq 0,-1,-2, \ldots, \quad \text { et } \gamma-\alpha \neq 1,2,3, \ldots \text {. }
$$

Soit $\theta$ un nombre réel tel que $-\frac{1}{2} \pi<\theta<0$ ou $0<\theta<\frac{1}{2} \pi$. Alors, on a

$$
\begin{aligned}
\lim _{\beta \rightarrow e^{i \theta} \infty} Q(\alpha, \beta, \gamma, z)= & \frac{\Gamma(\gamma) z^{\alpha-\gamma} e^{z}}{\Gamma(\gamma-\alpha) \Gamma(\alpha)\left(e^{2 \pi i(\gamma-\alpha)}-1\right)} \\
& \times \int_{e^{i \theta} \infty}^{(0+)} e^{-v} v^{\gamma-\alpha-1}\left(1-\frac{v}{z}\right)^{\alpha-1} d v
\end{aligned}
$$

et la convergence est localement uniforme sur le domaine $-\frac{1}{2} \pi<\arg z<\frac{5}{2} \pi$.

Démonstration. D'après le lemme 4.1 , on a

$$
\begin{aligned}
& Q(\alpha, \beta, \gamma, z)=\frac{\Gamma(\gamma) \Gamma(\alpha-\beta) \Gamma(\beta-\alpha+1) e^{\pi i(\gamma-\alpha)} \beta^{\gamma-\alpha} z^{\alpha-\gamma}}{\left(1-e^{2 \pi i(\gamma-\alpha)}\right)\left(1-e^{2 \pi i(\beta-\gamma)}\right) \Gamma(\gamma-\alpha) \Gamma(\alpha) \Gamma(\gamma-\beta) \Gamma(\beta-\gamma+1)} \\
& \quad \times\left(1+e^{-\pi i} \frac{z}{\beta}\right)^{\gamma-\alpha-\beta} \int^{(1+, 0+, 1-, 0-)} u^{\gamma-\alpha-1}(1-u)^{\beta-\gamma}\left(1-\frac{\beta u}{z}\right)^{\alpha-1} d u
\end{aligned}
$$

En substituant l'expression $u=\beta^{-1} v$ à l'intégrale définie du deuxième membre de l'égalité précédente, on a

$$
\begin{aligned}
\int^{(1+, 0+, 1-, 0-)} & u^{\gamma-\alpha-1}(1-u)^{\beta-\gamma}\left(1-\frac{\beta u}{z}\right)^{\alpha-1} d u \\
& =\beta^{\alpha-\gamma} \int^{(\beta+, 0+, \beta-, 0-)} v^{\gamma-\alpha-1}\left(1-\frac{v}{\beta}\right)^{\beta-\gamma}\left(1-\frac{v}{z}\right)^{\alpha-1} d v
\end{aligned}
$$


d'où

$$
\begin{aligned}
& Q(\alpha, \beta, \gamma, z)=\frac{\Gamma(\gamma) \Gamma(\alpha-\beta) \Gamma(\beta-\alpha+1) e^{\pi i(\gamma-\alpha)} z^{\alpha-\gamma}}{\left(1-e^{2 \pi i(\gamma-\alpha)}\right)\left(1-e^{2 \pi i(\beta-\gamma)}\right) \Gamma(\gamma-\alpha) \Gamma(\alpha) \Gamma(\gamma-\beta) \Gamma(\beta-\gamma+1)} \\
& \quad \times\left(1+e^{-\pi i} \frac{z}{\beta}\right)^{\gamma-\alpha-\beta} \int^{(\beta+, 0+, \beta-, 0-)} v^{\gamma-\alpha-1}\left(1-\frac{v}{\beta}\right)^{\beta-\gamma}\left(1-\frac{v}{z}\right)^{\alpha-1} d v .
\end{aligned}
$$

Posons $R=|\beta|$, et considérons $\left(1-R^{-1}\right) \beta$ comme point base du cycle de Pochhammer entourant $\beta$ et 0 ; on peut écrire

$$
\begin{aligned}
& \int^{(\beta+, 0+, \beta-, 0-)} v^{\gamma-\alpha-1}\left(1-\frac{v}{\beta}\right)^{\beta-\gamma}\left(1-\frac{v}{z}\right)^{\alpha-1} d v \\
& =\int_{\left(1-R^{-1}\right) \beta}^{(\beta+)} v^{\gamma-\alpha-1}\left(1-\frac{v}{\beta}\right)^{\beta-\gamma}\left(1-\frac{v}{z}\right)^{\alpha-1} d v \\
& +e^{2 \pi i(\beta-\gamma)} \int_{\left(1-R^{-1}\right) \beta}^{(0+)} v^{\gamma-\alpha-1}\left(1-\frac{v}{\beta}\right)^{\beta-\gamma}\left(1-\frac{v}{z}\right)^{\alpha-1} d v \\
& +e^{2 \pi i(\beta-\alpha)} \int_{\left(1-R^{-1}\right) \beta}^{(\beta-)} v^{\gamma-\alpha-1}\left(1-\frac{v}{\beta}\right)^{\beta-\gamma}\left(1-\frac{v}{z}\right)^{\alpha-1} d v \\
& +e^{2 \pi i(\gamma-\alpha)} \int_{\left(1-R^{-1}\right) \beta}^{(0-)} v^{\gamma-\alpha-1}\left(1-\frac{v}{\beta}\right)^{\beta-\gamma}\left(1-\frac{v}{z}\right)^{\alpha-1} d v \\
& =\left(1-e^{2 \pi i(\gamma-\alpha)}\right) \oint_{|v-\beta|=1} v^{\gamma-\alpha-1}\left(1-\frac{v}{\beta}\right)^{\beta-\gamma}\left(1-\frac{v}{z}\right)^{\alpha-1} d v \\
& +\left(e^{2 \pi i(\beta-\gamma)}-1\right) \int_{\left(1-R^{-1}\right) \beta}^{(0+)} v^{\gamma-\alpha-1}\left(1-\frac{v}{\beta}\right)^{\beta-\gamma}\left(1-\frac{v}{z}\right)^{\alpha-1} d v
\end{aligned}
$$

où $\oint_{|v-\beta|=1}$ désigne l'intégrale qui est prise le long du cercle $|v-\beta|=1$ dans le sens direct. En substituant le membre dernier de l'égalité précédente à l'intégrale définie dans (2.9), on a

$$
\begin{aligned}
Q(\alpha, \beta, \gamma, z)= & \frac{e^{\pi i(\gamma-\alpha)} \Gamma(\gamma) \Gamma(\alpha-\beta) \Gamma(\beta-\alpha+1) z^{\alpha-\gamma}}{\left(1-e^{2 \pi i(\beta-\gamma)}\right) \Gamma(\alpha) \Gamma(\gamma-\beta) \Gamma(\beta-\gamma+1) \Gamma(\gamma-\alpha)}\left(1+e^{-\pi i} \frac{z}{\beta}\right)^{\gamma-\alpha-\beta} \\
& \times \oint_{|v-\beta|=1} v^{\gamma-\alpha-1}\left(1-\frac{v}{\beta}\right)^{\beta-\gamma}\left(1-\frac{v}{z}\right)^{\alpha-1} d v \\
& +\frac{e^{\pi i(\gamma-\alpha)} \Gamma(\gamma) \Gamma(\alpha-\beta) \Gamma(\beta-\alpha+1) z^{\alpha-\gamma}}{\left(e^{2 \pi i(\gamma-\alpha)}-1\right) \Gamma(\alpha) \Gamma(\gamma-\alpha) \Gamma(\gamma-\beta) \Gamma(\beta-\gamma+1)}\left(1+e^{-\pi i} \frac{z}{\beta}\right)^{\gamma-\alpha-\beta} \\
& \times \int_{\left(1-R^{-1}\right) \beta}^{(0+)} v^{\gamma-\alpha-1}\left(1-\frac{v}{\beta}\right)^{\beta-\gamma}\left(1-\frac{v}{z}\right)^{\alpha-1} d v
\end{aligned}
$$

dont le deuxième membre est valable sous la condition (2.7). Pour prendre la limite $\lim _{\beta \rightarrow e^{i \theta} \infty} Q(\alpha, \beta, \gamma, z)$, il faut démontrer le 
Lemme 2.4. Soit $\theta$ un nombre réel tel que $-\frac{1}{2} \pi<\theta<0$ ou $0<\theta<\frac{1}{2} \pi$. On a:

(i) la quantité $\left|\frac{1}{1-e^{2 \pi i(\beta-\gamma)}}\right|$ est bornée pour $\beta \rightarrow e^{i \theta} \infty$;

(ii) $\lim _{\beta \rightarrow e^{i \theta} \infty}\left(1+e^{-\pi i} \frac{z}{\beta}\right)^{\gamma-\alpha-\beta}=e^{z}$;

(iii) $\lim _{\beta \rightarrow e^{i \theta} \infty} \frac{\Gamma(\beta-\alpha+1) \Gamma(\alpha-\beta)}{\Gamma(\gamma-\beta) \Gamma(\beta-\gamma+1)}=e^{\pi i(\alpha-\gamma)}$;

(iv) $\lim _{\beta \rightarrow e^{i \theta} \infty} \oint_{|v-\beta|=1} v^{\gamma-\alpha-1}\left(1-\frac{v}{\beta}\right)^{\beta-\gamma}\left(1-\frac{v}{z}\right)^{\alpha-1} d v=0$.

Démonstration. (i) et (ii) Evidente.

(iii) C'est le résultat immédiat des deux faits $\Gamma(\beta-\alpha+1) / \Gamma(\beta-\gamma+1) \sim \beta^{\alpha-\gamma}$ et $\Gamma(-\beta+\alpha) / \Gamma(-\beta+\gamma) \sim\left(e^{-\pi i} \beta\right)^{-\alpha+\gamma}$ d'après [9].

(iv) On pose $\beta=\operatorname{Re}^{i \theta}(R>0)$; on donne la nouvelle variable $\varphi(\theta-\pi \square \varphi \square \theta+\pi)$ par la formule $v=\beta+e^{i \varphi}$, d'où $d v=i e^{i \varphi} d \varphi$. Ainsi, on a

$$
\begin{aligned}
& \left|\oint_{|v-\beta|=1} v^{\gamma-\alpha-1}\left(1-\frac{v}{\beta}\right)^{\beta-\gamma}\left(1-\frac{v}{z}\right)^{\alpha-1} d v\right| \\
& =\left|\int_{\theta-\pi}^{\theta+\pi}\left(R e^{i \theta}+e^{i \varphi}\right)^{\gamma-\alpha-1}\left(\frac{e^{i(\varphi-\theta+\pi)}}{R}\right)^{R e^{i \theta}-\gamma}\left(1-\frac{R e^{i \theta}}{z}-\frac{e^{i \varphi}}{z}\right)^{\alpha-1} i e^{i \varphi} d \varphi\right| \\
& \square \int_{\theta-\pi}^{\theta+\pi}\left|\left(R e^{i \theta}+e^{i \varphi}\right)^{\gamma-\alpha-1}\left(\frac{e^{i(\varphi-\theta+\pi)}}{R}\right)^{-\gamma}\left(1-\frac{R e^{i \theta}}{z}-\frac{e^{i \varphi}}{z}\right)^{\alpha-1}\right| \times \\
& \times e^{-R[(\varphi-\theta+\pi) \sin \theta+\cos \theta \log R]} d \varphi .
\end{aligned}
$$

Ici, on a évidemment

$$
\lim _{R \rightarrow \infty}\left|\left(R e^{i \theta}+e^{i \varphi}\right)^{\gamma-\alpha-1}\left(\frac{e^{i(\varphi-\theta+\pi)}}{R}\right)^{-\gamma}\left(1-\frac{R e^{i \theta}}{z}-\frac{e^{i \varphi}}{z}\right)^{\alpha-1}\right| e^{-R[(\varphi-\theta+\pi) \sin \theta+\cos \theta \log R]}=0
$$

car $\lim _{R \rightarrow \infty} \cos \theta \log R=+\infty$. Par conséquant, le membre dernier de l'inégalité précédente tend vers 0 lorsque $R$ tend vers $\infty$, ce qui preuve la formule (iv) de ce lemme.

D'après le lemme 2.4, on aura aisément

$$
\lim _{\beta \rightarrow e^{i \theta} \infty} Q(\alpha, \beta, \gamma, z)=\frac{\Gamma(\gamma) z^{\alpha-\gamma} e^{z}}{\Gamma(\gamma-\alpha) \Gamma(\alpha)\left(e^{2 \pi i(\gamma-\alpha)}-1\right)} \int_{e^{i \theta} \infty}^{(0+)} e^{-v} v^{\gamma-\alpha-1}\left(1-\frac{v}{z}\right)^{\alpha-1} d v
$$

où le deuxième membre, fonction de $z$, vérifie l'équation (1.2). Comme $-\frac{1}{2} \pi<$ $\theta<0$ ou $0<\theta<\frac{1}{2} \pi$, l'intégrale définie dans le deuxième membre de (2.8) est certainement convergente $([3])$. De plus, on voit que le deuxième membre de (2.8) 
est valable pour $z$ tel que $-\frac{1}{2} \pi<\arg z<\frac{5}{2} \pi$, car $\theta<\arg z<\theta+2 \pi$. On voit aisément que la convergence de (2.8) est localement uniforme sur le domaine $-\frac{1}{2} \pi<$ $\arg z<\frac{5}{2} \pi$, si on regarde la démonstration du lemme 2.4. Ainsi la proposition 2.3 est démontrée.

L'unification des résultats des propositions 2.1 et 2.3 nous conduit au théorème suivant:

Théorème 2.5. Soient $\alpha, \gamma$ deux nombres complexes qui vérifient les conditions

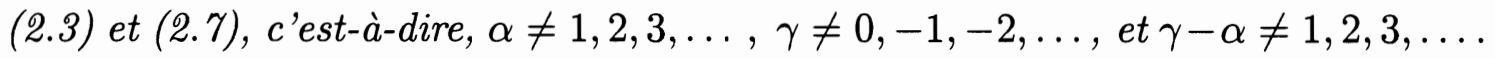
(i) Soit $\theta$ un nombre réel tel que $\frac{1}{2} \pi<\theta<\pi$ ou $\pi<\theta<\frac{3}{2} \pi$. En prenant la limite $\beta \rightarrow e^{i \theta} \infty$ pour la formule (2.1), on a

$$
\begin{aligned}
F(\alpha, \gamma, z)= & \frac{\Gamma(\gamma) z^{-\alpha}}{\Gamma(\gamma-\alpha) \Gamma(\alpha)\left(e^{\pi i \alpha}-e^{-\pi i \alpha}\right)} \int_{e^{i(\theta-\pi) \infty}}^{(0+)} e^{-v} v^{\alpha-1}\left(1+\frac{v}{z}\right)^{\gamma-\alpha-1} d v \\
& +\frac{\Gamma(\gamma) z^{\alpha-\gamma} e^{z}}{\Gamma(\alpha) \Gamma(\gamma-\alpha)\left(e^{2 \pi i(\gamma-\alpha)}-1\right)} \int_{e^{i(\theta-\pi) \infty}}^{(0+)} e^{-v} v^{\gamma-\alpha-1}\left(1-\frac{v}{z}\right)^{\alpha-1} d v
\end{aligned}
$$

et la convergence est localement uniforme sur le domaine $-\frac{1}{2} \pi<\arg z<\frac{3}{2} \pi$.

(ii) Soit $\theta$ un nombre réel tel que $-\frac{1}{2} \pi<\theta<0$ ou $0<\theta<\frac{1}{2} \pi$. En prenant la limite $\beta \rightarrow e^{i \theta} \infty$ pour la formule (2.1), on a

$$
\begin{aligned}
F(\alpha, \gamma, z)= & \frac{\Gamma(\gamma) z^{-\alpha}}{\Gamma(\gamma-\alpha) \Gamma(\alpha)\left(e^{\pi i \alpha}-e^{-\pi i \alpha}\right)} \int_{e^{i \theta} \infty}^{(0+)} e^{-v} v^{\alpha-1}\left(1+\frac{v}{z}\right)^{\gamma-\alpha-1} d v \\
& +\frac{\Gamma(\gamma) z^{\alpha-\gamma} e^{z}}{\Gamma(\alpha) \Gamma(\gamma-\alpha)\left(e^{2 \pi i(\gamma-\alpha)}-1\right)} \int_{e^{i \theta} \infty}^{(0+)} e^{-v} v^{\gamma-\alpha-1}\left(1-\frac{v}{z}\right)^{\alpha-1} d v,
\end{aligned}
$$

et la convergence est localement uniforme sur le domaine $-\frac{1}{2} \pi<\arg z<\frac{3}{2} \pi$.

Démonstration. Notons que chacune des fonctions $P(\alpha, \beta, \gamma, z)$ et $Q(\alpha, \beta, \gamma, z)$ est une relation linéaire entre les fonctions $F(\alpha, \beta, \gamma, z / \beta)$ et $(z / \beta)^{1-\gamma} F(1+\alpha-\gamma, 1+$ $\beta-\gamma, 2-\gamma, z / \beta)$. Pour cette relation on peut prendre la limite $\beta \rightarrow e^{i \theta} \infty$ pour tout $\theta \in \mathbf{R}$. D'où suit aussitôt le théorème.

Remarque 2.1. Il est facile à déduire de (2.9) ou de (2.10) le développement asymptotique en $\infty$ pour la fonction hypergéométrique confluente $F(\alpha, \gamma, z)$. En effet, selon Inui ([3]), on aura aussitôt de (2.9) ou de (2.10) le développement asymptotique

$$
\begin{aligned}
F(\alpha, \gamma, z) \sim & \frac{\Gamma(\gamma)}{\Gamma(\gamma-\alpha)}\left(e^{-\pi i} z\right)^{-\alpha} \sum_{n=0}^{\infty} \frac{(-1)^{n}(\alpha)_{n}(\alpha-\gamma+1)_{n}}{n !} \frac{1}{z^{n}} \\
& +\frac{\Gamma(\gamma)}{\Gamma(\alpha)} z^{\alpha-\gamma} e^{z} \sum_{n=0}^{\infty} \frac{(1-\alpha)_{n}(\gamma-\alpha)_{n}}{n !} \frac{1}{z^{n}}
\end{aligned}
$$


qui est valable pour le voisinage sectoriel de $\infty$ défini par $-\frac{1}{2} \pi<\arg z<\frac{3}{2} \pi$.

Remarque 2.2. Posons

$$
G_{1}(\alpha, \gamma, z)=\frac{\Gamma(\gamma) z^{-\alpha}}{\Gamma(\gamma-\alpha) \Gamma(\alpha)\left(e^{\pi i \alpha}-e^{-\pi i \alpha}\right)} \int_{e^{i(\theta-\pi) \infty}}^{(0+)} e^{-v} v^{\alpha-1}\left(1+\frac{v}{z}\right)^{\gamma-\alpha-1} d v
$$

et

$$
G_{2}(\alpha, \gamma, z)=\frac{\Gamma(\gamma) z^{\alpha-\gamma} e^{z}}{\Gamma(\alpha) \Gamma(\gamma-\alpha)\left(e^{2 \pi i(\gamma-\alpha)}-1\right)} \int_{e^{i(\theta-\pi) \infty}}^{(0+)} e^{-v} v^{\gamma-\alpha-1}\left(1-\frac{v}{z}\right)^{\alpha-1} d v
$$

Alors, on voit aisément la formule suivante:

$$
G_{2}(\alpha, \gamma, z)=e^{2 \pi i(\alpha-\gamma)} e^{z} G_{1}\left(\gamma-\alpha, \gamma, e^{-\pi i} z\right)
$$

\section{$\S 3$ Dégénérescence de la formule de connexion entre 0 et 1}

Dans ce paragraphe, nous étudierons la dégénérescence de la formule de connexion entre 0 et 1 . Nous commençons par la formule suivante:

$$
\begin{aligned}
& F(\alpha, \beta, \gamma, z)=\frac{\Gamma(\gamma) \Gamma(\gamma-\alpha-\beta)}{\Gamma(\gamma-\beta) \Gamma(\gamma-\alpha)} F(\alpha, \beta, 1+\alpha+\beta-\gamma, 1-z)+ \\
& \quad+\frac{\Gamma(\gamma) \Gamma(\alpha+\beta-\gamma)}{\Gamma(\alpha) \Gamma(\beta)}\left(1+e^{-\pi i} z\right)^{\gamma-\alpha-\beta} F(\gamma-\alpha, \gamma-\beta, 1+\gamma-\alpha-\beta, 1-z),
\end{aligned}
$$

qui est valable pour trois nombres complexes $\alpha, \beta, \gamma$ tels que $\gamma \neq 0,-1,-2, \ldots$, et $\alpha+\beta-\gamma \notin \mathbf{Z}$. Comme $F(\alpha, \beta, 1+\alpha+\beta-\gamma, 1-z)=z^{-\alpha} F(\alpha-\gamma+1, \alpha, \alpha+$ $\left.\beta-\gamma+1,(z-1) z^{-1}\right)$ et $\left(1+e^{-\pi i} z\right)^{\gamma-\alpha-\beta} F(\gamma-\alpha, \gamma-\beta, 1+\gamma-\alpha-\beta, 1-z)=$ $z^{\alpha-\gamma}\left(1+e^{-\pi i} z\right)^{\gamma-\alpha-\beta} F\left(1-\alpha, \gamma-\alpha, 1+\gamma-\alpha-\beta,(z-1) z^{-1}\right)$ d'après Kummer $([4])$, la formule $(3.1)$ se change en

$$
\begin{aligned}
& F(\alpha, \beta, \gamma, z)=\frac{\Gamma(\gamma) \Gamma(\gamma-\alpha-\beta)}{\Gamma(\gamma-\beta) \Gamma(\gamma-\alpha)} z^{-\alpha} F\left(\alpha-\gamma+1, \alpha, \alpha+\beta-\gamma+1, \frac{z-1}{z}\right)+ \\
& +\frac{\Gamma(\gamma) \Gamma(\alpha+\beta-\gamma)}{\Gamma(\alpha) \Gamma(\beta)} z^{\alpha-\gamma}\left(1+e^{-\pi i} z\right)^{\gamma-\alpha-\beta} F\left(1-\alpha, \gamma-\alpha, 1+\gamma-\alpha-\beta, \frac{z-1}{z}\right)
\end{aligned}
$$

Remplaçons la variable $z$ dans l'égalité précédente par $z / \beta$, où $\beta \neq 0$; on a alors

$$
\begin{aligned}
& F\left(\alpha, \beta, \gamma, \frac{z}{\beta}\right) \\
& =\frac{\Gamma(\gamma) \Gamma(\gamma-\alpha-\beta)}{\Gamma(\gamma-\beta) \Gamma(\gamma-\alpha)} \beta^{\alpha} z^{-\alpha} F\left(\alpha-\gamma+1, \alpha, \alpha+\beta-\gamma+1, \beta \frac{(z / \beta)-1}{z}\right)+ \\
& +\frac{\Gamma(\gamma) \Gamma(\alpha+\beta-\gamma)}{\Gamma(\alpha) \Gamma(\beta)} \beta^{\gamma-\alpha} z^{\alpha-\gamma}\left(1+e^{-\pi i} \frac{z}{\beta}\right)^{\gamma-\alpha-\beta} F\left(1-\alpha, \gamma-\alpha, 1+\gamma-\alpha-\beta, \beta \frac{(z / \beta)-1}{z}\right)
\end{aligned}
$$


Soit $P(\alpha, \beta, \gamma, z)$ une détermination du premier terme du deuxième membre de (3.2), telle que, si $0<z<1, \arg z=0$ :

$$
\begin{aligned}
& P(\alpha, \beta, \gamma, z) \\
& \quad=\frac{\Gamma(\gamma) \Gamma(\gamma-\alpha-\beta)}{\Gamma(\gamma-\beta) \Gamma(\gamma-\alpha)} \beta^{\alpha} z^{-\alpha} F\left(\alpha-\gamma+1, \alpha, \alpha+\beta-\gamma+1, \beta \frac{(z / \beta)-1}{z}\right) .
\end{aligned}
$$

Nous preuverons la

Proposition 3.1. Soient $\alpha, \gamma$ deux nombres complexes tels que

$$
\alpha \neq 1,2,3, \ldots, \quad \text { et } \gamma \neq 0,-1,-2, \ldots
$$

Soit $\theta$ un nombre réel tel que $-\frac{1}{2} \pi<\theta<0$ ou $0<\theta<\frac{1}{2} \pi$. Alors, on a

$$
\begin{aligned}
\lim _{\beta \rightarrow e^{i \theta} \infty} P(\alpha, \beta, \gamma, z)= & \frac{\Gamma(\gamma) z^{-\alpha}}{\Gamma(\gamma-\alpha) \Gamma(\alpha)\left(e^{\pi i \alpha}-e^{-\pi i \alpha}\right)} \\
& \times \int_{e^{i \theta} \infty}^{(0+)} e^{-v} v^{\alpha-1}\left(1+\frac{v}{z}\right)^{\gamma-\alpha-1} d v
\end{aligned}
$$

et la convergence est localement uniforme sur le domaine $-\frac{3}{2} \pi<\arg z<\frac{3}{2} \pi$.

Démonstration. D'après le lemme 4.1 , on a

$$
\begin{aligned}
P(\alpha, \beta, \gamma, z)= & \frac{\Gamma(\gamma) \Gamma(\gamma-\alpha-\beta) \Gamma(\alpha+\beta-\gamma+1) \beta^{\alpha} z^{-\alpha}}{\left(1-e^{2 \pi i \alpha}\right)\left(1-e^{2 \pi i(\beta-\gamma)}\right) \Gamma(\alpha) \Gamma(\gamma-\alpha) \Gamma(\beta-\gamma+1) \Gamma(\gamma-\beta)} \\
& \times \int^{(1+, 0+, 1-, 0-)} u^{\alpha-1}(1-u)^{\beta-\gamma}\left(1-\beta \frac{(z / \beta)-1}{z} u\right)^{\gamma-\alpha-1} d u
\end{aligned}
$$

En substituant l'expression $u=\beta^{-1} v$ à l'intégrale définie du deuxième membre de l'égalité précédente, on a

$$
\begin{aligned}
\int^{(1+, 0+, 1-, 0-)} & u^{\alpha-1}(1-u)^{\beta-\gamma}\left(1-\beta \frac{(z / \beta)-1}{z} u\right)^{\gamma-\alpha-1} d u \\
= & \beta^{-\alpha} \int^{(\beta+, 0+, \beta-, 0-)} v^{\alpha-1}\left(1-\frac{v}{\beta}\right)^{\beta-\gamma}\left(1-\frac{(z / \beta)-1}{z} v\right)^{\gamma-\alpha-1} d v
\end{aligned}
$$

d'où

$$
\begin{aligned}
P(\alpha, \beta, \gamma, z)= & \frac{\Gamma(\gamma) \Gamma(\gamma-\alpha-\beta) \Gamma(\alpha+\beta-\gamma+1) z^{-\alpha}}{\left(1-e^{2 \pi i \alpha}\right)\left(1-e^{2 \pi i(\beta-\gamma)}\right) \Gamma(\alpha) \Gamma(\gamma-\alpha) \Gamma(\beta-\gamma+1) \Gamma(\gamma-\beta)} \\
& \times \int^{(\beta+, 0+, \beta-, 0-)} v^{\alpha-1}\left(1-\frac{v}{\beta}\right)^{\beta-\gamma}\left(1-\frac{(z / \beta)-1}{z} v\right)^{\gamma-\alpha-1} d v
\end{aligned}
$$


Posons $R=|\beta|$, et considérons $\left(1-R^{-1}\right) \beta$ comme point base du cycle de Pochhammer entourant $\beta$ et 0 ; on peut écrire

$$
\begin{aligned}
& \int_{(1-, 0+, \beta-, 0-)} v^{\alpha-1}\left(1-\frac{v}{\beta}\right)^{\beta-\gamma}\left(1-\frac{(z / \beta)-1}{z} v\right)^{\gamma-\alpha-1} d v \\
& =\int_{\left(1-R^{-1}\right) \beta}^{(\beta+)} v^{\alpha-1}\left(1-\frac{v}{\beta}\right)^{\beta-\gamma}\left(1-\frac{(z / \beta)-1}{z} v\right)^{\gamma-\alpha-1} d v \\
& +e^{2 \pi i(\beta-\gamma)} \int_{\left(1-R^{-1}\right) \beta}^{(0+)} v^{\alpha-1}\left(1-\frac{v}{\beta}\right)^{\beta-\gamma}\left(1-\frac{(z / \beta)-1}{z} v\right)^{\gamma-\alpha-1} d v \\
& +e^{2 \pi i(\alpha+\beta-\gamma)} \int_{\left(1-R^{-1}\right) \beta}^{(\beta-)} v^{\alpha-1}\left(1-\frac{v}{\beta}\right)^{\beta-\gamma}\left(1-\frac{(z / \beta)-1}{z} v\right)^{\gamma-\alpha-1} d v \\
& +e^{2 \pi i \alpha} \int_{\left(1-R^{-1}\right) \beta}^{(0-)} v^{\alpha-1}\left(1-\frac{v}{\beta}\right)^{\beta-\gamma}\left(1-\frac{(z / \beta)-1}{z} v\right)^{\gamma-\alpha-1} d v \\
& =\left(1-e^{2 \pi i \alpha}\right) \oint_{|v-\beta|=1} v^{\alpha-1}\left(1-\frac{v}{\beta}\right)^{\beta-\gamma}\left(1-\frac{(z / \beta)-1}{z} v\right)^{\gamma-\alpha-1} d v \\
& +\left(e^{2 \pi i(\beta-\gamma)}-1\right) \int_{\left(1-R^{-1}\right) \beta}^{(0+)} v^{\alpha-1}\left(1-\frac{v}{\beta}\right)^{\beta-\gamma}\left(1-\frac{(z / \beta)-1}{z} v\right)^{\gamma-\alpha-1} d v,
\end{aligned}
$$

où $\oint_{|v-\beta|=1}$ désigne l'intégrale qui est prise le long du cercle $|v-\beta|=1$ dans le sens direct. En substituant le membre dernier de l'égalité précédente à l'intégrale définie de (3.6), on a

$$
\begin{aligned}
P(\alpha, \beta, & \gamma, z) \\
= & \frac{\Gamma(\gamma) \Gamma(\gamma-\alpha-\beta) \Gamma(\alpha+\beta-\gamma+1) z^{-\alpha}}{\left(1-e^{2 \pi i(\beta-\gamma)}\right) \Gamma(\alpha) \Gamma(\gamma-\alpha) \Gamma(\gamma-\beta) \Gamma(\beta-\gamma+1)} \times \\
& \times \oint_{|v-\beta|=1} v^{\alpha-1}\left(1-\frac{v}{\beta}\right)^{\beta-\gamma}\left(1-\frac{(z / \beta)-1}{z} v\right)^{\gamma-\alpha-1} d v+ \\
+ & \frac{\Gamma(\gamma) \Gamma(\gamma-\alpha-\beta) \Gamma(\alpha+\beta-\gamma+1) z^{-\alpha}}{\left(e^{2 \pi i \alpha}-1\right) \Gamma(\alpha) \Gamma(\gamma-\alpha) \Gamma(\gamma-\beta) \Gamma(\beta-\gamma+1)} \times \\
& \times \int_{\left(1-R^{-1}\right) \beta}^{(0+)} v^{\alpha-1}\left(1-\frac{v}{\beta}\right)^{\beta-\gamma}\left(1-\frac{(z / \beta)-1}{z} v\right)^{\gamma-\alpha-1} d v,
\end{aligned}
$$

dont le deuxième membre est valable sous la condition (3.4). Pour prendre la limite $\lim _{\beta \rightarrow e^{i \theta}} P(\alpha, \beta, \gamma, z)$, il faut démontrer le

Lemme 3.2. Soit $\theta$ un nombre réel tel que $-\frac{1}{2} \pi<\theta<0$ ou $0<\theta<\frac{1}{2} \pi$. On a:

(i) $\lim _{\beta \rightarrow e^{i \theta} \infty} \frac{\Gamma(\gamma-\beta-\alpha) \Gamma(\alpha+\beta-\gamma+1)}{\Gamma(\gamma-\beta) \Gamma(\beta-\gamma+1)}=e^{\pi i \alpha}$;

(ii) $\lim _{\beta \rightarrow e^{i \theta} \infty} \oint_{|v-\beta|=1} v^{\alpha-1}\left(1-\frac{v}{\beta}\right)^{\beta-\gamma}\left(1-\frac{(z / \beta)-1}{z} v\right)^{\gamma-\alpha-1} d v=0$. 
Démonstration. (i) C'est le résultat immédiat des deux faits $\Gamma(\alpha+\beta-\gamma+1) / \Gamma(\beta-$ $\gamma+1) \sim \beta^{-\alpha}$ et $\Gamma(\gamma-\alpha-\beta) / \Gamma(\gamma-\beta) \sim\left(e^{\pi i} \beta\right)^{\alpha}$ d'après [9].

(ii) On pose $\beta=\operatorname{Re}^{i \theta}(R>0)$; on donne la nouvelle variable $\varphi(\theta-\pi \square \varphi \square \theta+\pi)$ par la formule $v-\beta=e^{i \varphi}$, d'où $d v=i e^{i \varphi} d \varphi$. Ainsi, on a

$$
\begin{gathered}
\left|\oint_{|v-\beta|=1} v^{\alpha-1}\left(1-\frac{v}{\beta}\right)^{\beta-\gamma}\left(1-\frac{(z / \beta)-1}{z} v\right)^{\gamma-\alpha-1} d v\right| \\
=\left|\int_{\theta-\pi}^{\theta+\pi}\left(R e^{i \theta}+e^{i \varphi}\right)^{\alpha-1}\left(\frac{e^{i(\varphi-\theta+\pi)}}{R}\right)^{R e^{i \theta}-\gamma}\left(1-\frac{(z / \beta)-1}{z}\left(R e^{i \theta}+e^{i \varphi}\right)\right)^{\gamma-\alpha-1} i e^{i \varphi} d \varphi\right| \\
\square \int_{\theta-\pi}^{\theta+\pi}\left|\left(R e^{i \theta}+e^{i \varphi}\right)^{\alpha-1}\left(\frac{e^{i(\varphi-\theta+\pi)}}{R}\right)^{-\gamma}\left(1-\frac{(z / \beta)-1}{z}\left(R e^{i \theta}+e^{i \varphi}\right)\right)^{\gamma-\alpha-1}\right| \times \\
\times e^{-R[(\varphi-\theta+\pi) \sin \theta+\cos \theta \log R] d \varphi .}
\end{gathered}
$$

Ici, on a évidemment

$$
\begin{aligned}
\lim _{R \rightarrow \infty} \mid\left(R e^{i \theta}+e^{i \varphi}\right)^{\alpha-1} & \left(\frac{e^{i(\varphi-\theta+\pi)}}{R}\right)^{-\gamma}\left(1-\frac{(z / \beta)-1}{z}\left(R e^{i \theta}+e^{i \varphi}\right)\right)^{\gamma-\alpha-1} \mid \times \\
& \times e^{-R[(\varphi-\theta+\pi) \sin \theta+\cos \theta \log R]=0,}
\end{aligned}
$$

car $\lim _{R \rightarrow \infty} \cos \theta \log R=+\infty$. Par conséquant, le membre dernier de l'inégalité précédente tend vers 0 lorsque $R$ tend vers $\infty$, ce qui preuve la formule (ii) de ce lemme.

D'après le lemme 3.2 , on aura aisément

$$
\begin{aligned}
\lim _{\beta \rightarrow e^{i \theta} \infty} P(\alpha, \beta, \gamma, z)= & \frac{\Gamma(\gamma) z^{-\alpha}}{\Gamma(\gamma-\alpha) \Gamma(\alpha)\left(e^{\pi i \alpha}-e^{-\pi i \alpha}\right)} \\
& \times \int_{e^{i \theta} \infty}^{(0+)} e^{-v} v^{\alpha-1}\left(1+\frac{v}{z}\right)^{\gamma-\alpha-1} d v
\end{aligned}
$$

où le deuxième membre, fonction de $z$, vérifie l'équation (1.2). Comme $-\frac{1}{2} \pi<$ $\theta<0$ ou $0<\theta<\frac{1}{2} \pi$, l'intégrale définie dans le deuxième membre de (3.5) est certainement convergente (voir [3]). De plus, on voit que le deuxième membre de (3.5) est valable pour $z$ tel que $-\frac{3}{2} \pi<\arg z<\frac{3}{2} \pi$, $\operatorname{car} \theta<\arg \left(e^{\pi i} z\right)<\theta+2 \pi$, i.e., $\theta-\pi<\arg z<\theta+\pi$. On voit aisément que la convergence de (3.5) est localement uniforme sur le domaine $-\frac{3}{2} \pi<\arg z<\frac{3}{2} \pi$, si on regarde la démonstration du lemme 3.2. Ainsi la proposition 3.1 est démontrée.

Soit $Q(\alpha, \beta, \gamma, z)$ une détermination du deuxième terme du deuxième membre de 
(3.2), telle que, si $0<z<1, \arg z=0$ :

$$
\begin{aligned}
Q(\alpha, \beta, \gamma, z)= & \frac{\Gamma(\gamma) \Gamma(\alpha+\beta-\gamma)}{\Gamma(\alpha) \Gamma(\beta)} \beta^{\gamma-\alpha} z^{\alpha-\gamma}\left(1+e^{-\pi i} \frac{z}{\beta}\right)^{\gamma-\alpha-\beta} \times \\
& \times F\left(1-\alpha, \gamma-\alpha, 1+\gamma-\alpha-\beta, \beta \frac{(z / \beta)-1}{z}\right) .
\end{aligned}
$$

Nous preuverons la

Proposition 3.3. Soient $\alpha, \gamma$ deux nombres complexes tels que

$$
\gamma \neq 0,-1,-2, \ldots, \quad \text { et } \gamma-\alpha \neq 1,2,3, \ldots
$$

Soit $\theta$ un nombre réel tel que $\frac{1}{2} \pi<\theta<\pi$ ou $\pi<\theta<\frac{3}{2} \pi$. Alors, on a

$$
\begin{array}{rl}
\lim _{\beta \rightarrow e^{i \theta} \infty} Q & Q(\alpha, \beta, \gamma, z) \\
& =\frac{\Gamma(\gamma) z^{\alpha-\gamma} e^{z}}{\Gamma(\gamma-\alpha) \Gamma(\alpha)\left(e^{2 \pi i(\gamma-\alpha)}-1\right)} \int_{e^{i(\theta-\pi) \infty}}^{(0+)} e^{-v} v^{\gamma-\alpha-1}\left(1-\frac{v}{z}\right)^{\alpha-1} d v
\end{array}
$$

et la convergence est localement uniforme sur le domaine $-\frac{1}{2} \pi<\arg z<\frac{5}{2} \pi$.

Démonstration. D'après le lemme 4.1, on a

$$
\begin{aligned}
Q(\alpha, \beta, \gamma, z)= & \frac{\Gamma(\gamma) \Gamma(\alpha+\beta-\gamma) \Gamma(1+\gamma-\alpha-\beta) \beta^{\gamma-\alpha} z^{\alpha-\gamma}}{\left(1-e^{2 \pi i(\gamma-\alpha)}\right)\left(1-e^{-2 \pi i \beta}\right) \Gamma(\gamma-\alpha) \Gamma(1-\beta) \Gamma(\alpha) \Gamma(\beta)}\left(1+e^{-\pi i} \frac{z}{\beta}\right)^{\gamma-\alpha-\beta} \times \\
& \times \int^{(1+, 0+, 1-, 0-)} u^{\gamma-\alpha-1}(1-u)^{-\beta}\left(1-\beta \frac{(z / \beta)-1}{z} u\right)^{\alpha-1} d u
\end{aligned}
$$

En substituant l'expression $u=e^{\pi i} \beta^{-1} v$ à l'intégrale définie du deuxième membre de l'égalité précédente, on a

$$
\begin{aligned}
& \int^{(1+, 0+, 1-, 0-)} u^{\gamma-\alpha-1}(1-u)^{-\beta}\left(1-\beta \frac{(z / \beta)-1}{z} u\right)^{\alpha-1} d u \\
= & e^{\pi i(\gamma-\alpha)} \beta^{\alpha-\gamma} \int^{\left(e^{-\pi i} \beta+, 0+, e^{-\pi i} \beta-, 0-\right)} v^{\gamma-\alpha-1}\left(1+\frac{v}{\beta}\right)^{-\beta}\left(1+\frac{(z / \beta)-1}{z} v\right)^{\alpha-1} d v
\end{aligned}
$$

d'où

$$
\begin{aligned}
Q(\alpha, \beta, \gamma, z)= & \frac{\Gamma(\gamma) \Gamma(\alpha+\beta-\gamma) \Gamma(1+\gamma-\alpha-\beta) e^{\pi i(\gamma-\alpha)} z^{\alpha-\gamma}}{\left(1-e^{2 \pi i(\gamma-\alpha)}\right)\left(1-e^{-2 \pi i \beta}\right) \Gamma(\gamma-\alpha) \Gamma(1-\beta) \Gamma(\alpha) \Gamma(\beta)}\left(1+e^{-\pi i} \frac{z}{\beta}\right)^{\gamma-\alpha-\beta} \times \\
& \times \int^{\left(e^{-\pi i} \beta+, 0+, e^{-\pi i} \beta-, 0-\right)} v^{\gamma-\alpha-1}\left(1+\frac{v}{\beta}\right)^{-\beta}\left(1+\frac{(z / \beta)-1}{z} v\right)^{\alpha-1} d v
\end{aligned}
$$


Posons $R=|\beta|$, et considérons $\left(1-R^{-1}\right) e^{-\pi i} \beta$ comme point base du cycle de Pochhammer entourant $e^{-\pi i} \beta$ et 0 ; on peut écrire

$$
\begin{aligned}
& \int^{\left(e^{-\pi i} \beta+, 0+, e^{-\pi i} \beta-, 0-\right)} v^{\gamma-\alpha-1}\left(1+\frac{v}{\beta}\right)^{-\beta}\left(1+\frac{(z / \beta)-1}{z} v\right)^{\alpha-1} d v \\
& =\int_{\left(1-R^{-1}\right) e^{-\pi i} \beta}^{\left(e^{-\pi i} \beta+\right)} v^{\gamma-\alpha-1}\left(1+\frac{v}{\beta}\right)^{-\beta}\left(1+\frac{(z / \beta)-1}{z} v\right)^{\alpha-1} d v \\
& +e^{-2 \pi i \beta} \int_{\left(1-R^{-1}\right) e^{-\pi i} \beta}^{(0+)} v^{\gamma-\alpha-1}\left(1+\frac{v}{\beta}\right)^{-\beta}\left(1+\frac{(z / \beta)-1}{z} v\right)^{\alpha-1} d v \\
& +e^{2 \pi i(\gamma-\alpha-\beta)} \int_{\left(1-R^{-1}\right) e^{-\pi i} \beta}^{\left(e^{-\pi i} \beta-\right)} v^{\gamma-\alpha-1}\left(1+\frac{v}{\beta}\right)^{-\beta}\left(1+\frac{(z / \beta)-1}{z} v\right)^{\alpha-1} d v \\
& +e^{2 \pi i(\gamma-\alpha)} \int_{\left(1-R^{-1}\right) e^{-\pi i} \beta}^{(0-)} v^{\gamma-\alpha-1}\left(1+\frac{v}{\beta}\right)^{-\beta}\left(1+\frac{(z / \beta)-1}{z} v\right)^{\alpha-1} d v \\
& =\left(1-e^{2 \pi i(\gamma-\alpha)} \oint_{\left|v-e^{-\pi i} \beta\right|=1} v^{\gamma-\alpha-1}\left(1+\frac{v}{\beta}\right)^{-\beta}\left(1+\frac{(z / \beta)-1}{z} v\right)^{\alpha-1} d v\right. \\
& +\left(e^{-2 \pi i \beta}-1\right) \int_{\left(1-R^{-1}\right) e^{-\pi i} \beta}^{(0+)} v^{\gamma-\alpha-1}\left(1+\frac{v}{\beta}\right)^{-\beta}\left(1+\frac{(z / \beta)-1}{z} v\right)^{\alpha-1} d v
\end{aligned}
$$

où $\oint_{\left|v-e^{-\pi i} \beta\right|=1}$ désigne l'intégrale qui est prise le long du cercle $\left|v-e^{-\pi i} \beta\right|=1$ dans le sens direct. En substituant le membre dernier de l'égalité précédente à l'intégrale définie de $(3.10)$, on a

$$
\begin{aligned}
& Q(\alpha, \beta, \gamma, z) \\
& =\frac{e^{\pi i(\gamma-\alpha)} \Gamma(\gamma) \Gamma(\alpha+\beta-\gamma) \Gamma(1+\gamma-\alpha-\beta)}{\left(1-e^{-2 \pi i \beta}\right) \Gamma(\alpha) \Gamma(\beta) \Gamma(\gamma-\alpha) \Gamma(1-\beta)} z^{\alpha-\gamma}\left(1+e^{-\pi i} \frac{z}{\beta}\right)^{\gamma-\alpha-\beta} \\
& \quad \times \oint_{\left|v-e^{-\pi i} \beta\right|=1} v^{\gamma-\alpha-1}\left(1+\frac{v}{\beta}\right)^{-\beta}\left(1+\frac{(z / \beta)-1}{z} v\right)^{\alpha-1} d v \\
& +\frac{\Gamma(\gamma) \Gamma(\alpha+\beta-\gamma) \Gamma(1+\gamma-\alpha-\beta) z^{\alpha-\gamma}}{\left(e^{\pi i(\gamma-\alpha)}-e^{\pi i(\alpha-\gamma)}\right) \Gamma(\alpha) \Gamma(\gamma-\alpha) \Gamma(\beta) \Gamma(1-\beta)}\left(1+e^{-\pi i} \frac{z}{\beta}\right)^{\gamma-\alpha-\beta} \\
& \quad \times \int_{\left(1-R^{-1}\right) e^{-\pi i} \beta}^{(0+)} v^{\gamma-\alpha-1}\left(1+\frac{v}{\beta}\right)^{-\beta}\left(1+\frac{(z / \beta)-1}{z} v\right)^{\alpha-1} d v,
\end{aligned}
$$

dont le deuxième membre est valable sous la condition (3.8). Pour prendre la limite $\lim _{R \rightarrow e^{i \theta} \infty} Q(\alpha, \beta, \gamma, z)$, il faut démontrer le

Lemme 3.4. Soit $\theta$ un nombre réel tel que $\frac{1}{2} \pi<\theta<\pi$ ou $\pi<\theta<\frac{3}{2} \pi$. On a:

(i) $\lim _{\beta \rightarrow e^{i \theta} \infty} \frac{\Gamma(\alpha+\beta-\gamma) \Gamma(1+\gamma-\alpha-\beta)}{\Gamma(\beta) \Gamma(1-\beta)}=e^{\pi i(\alpha-\gamma)}$;

(ii) $\lim _{\beta \rightarrow e^{i \theta} \infty} \oint_{\left|v-e^{-\pi i} \beta\right|=1} v^{\gamma-\alpha-1}\left(1+\frac{v}{\beta}\right)^{-\beta}\left(1+\frac{(z / \beta)-1}{z} v\right)^{\alpha-1} d v=0$. 
Démonstration. (i) C'est le résultat immédiat des deux faits $\Gamma(\alpha+\beta-\gamma) / \Gamma(\beta) \sim$ $\beta^{\gamma-\alpha}$ et $\Gamma(-\beta+1+\gamma-\alpha) / \Gamma(-\beta+1) \sim\left(e^{\pi i} \beta\right)^{\alpha-\gamma}$ d'après [9].

(ii) On pose $\beta=\operatorname{Re}^{i \theta}(R>0)$; on donne la nouvelle variable $\varphi(\theta-2 \pi \square \varphi \square \theta)$ par la formule $v-e^{-\pi i} \beta=e^{i \varphi}$, d'où $d v=i e^{i \varphi} d \varphi$. Ainsi, on a

$$
\begin{aligned}
& \left|\oint_{\left|v-e^{-\pi i} \beta\right|=1} v^{\gamma-\alpha-1}\left(1+\frac{v}{\beta}\right)^{-\beta}\left(1+\frac{(z / \beta)-1}{z} v\right)^{\alpha-1} d v\right| \\
& =\left|\int_{\theta-2 \pi}^{\theta}\left(R e^{i(\theta-\pi)}+e^{i \varphi}\right)^{\gamma-\alpha-1}\left(\frac{e^{i(\varphi-\theta)}}{R}\right)^{-R e^{i \theta}}\left(1+\frac{(z / \beta)-1}{z}\left(e^{-\pi i} \beta+e^{i \varphi}\right)\right)^{\alpha-1} i e^{i \varphi} d \varphi\right| \\
& \square \int_{\theta-2 \pi}^{\theta}\left|\left(R e^{i(\theta-\pi)}+e^{i \varphi}\right)^{\gamma-\alpha-1}\left(1+\frac{(z / \beta)-1}{z}\left(e^{-\pi i} \beta+e^{i \varphi}\right)\right)^{\alpha-1}\right| \times \\
& \times e^{R[(\varphi-\theta) \sin \theta+\cos \theta \log R]} d \varphi .
\end{aligned}
$$

Ici, on a évidemment

$$
\begin{aligned}
\lim _{R \rightarrow \infty} \mid\left(R e^{i(\theta-\pi)}+e^{i \varphi}\right)^{\gamma-\alpha-1} & \left(1+\frac{(z / \beta)-1}{z}\left(e^{-\pi i} \beta+e^{i \varphi}\right)\right)^{\alpha-1} \mid \times \\
& \times e^{R[(\varphi-\theta) \sin \theta+\cos \theta \log R]=0,}
\end{aligned}
$$

car $\lim _{R \rightarrow \infty} \cos \theta \log R=-\infty$. Par conséquent, le membre dernier de l'inégalité précédente tend vers 0 lorsque $R$ tend vers $\infty$, ce qui preuve la formule (ii) de ce lemme.

D'après le lemme 3.4 , on aura aisément

$$
\begin{aligned}
\lim _{\beta \rightarrow e^{i \theta} \infty} Q(\alpha, \beta, \gamma, z)= & \frac{\Gamma(\gamma) z^{\alpha-\gamma} e^{z}}{\Gamma(\gamma-\alpha) \Gamma(\alpha)\left(e^{2 \pi i(\gamma-\alpha)}-1\right)} \\
& \times \int_{e^{i(\theta-\pi)} \infty}^{(0+)} e^{-v} v^{\gamma-\alpha-1}\left(1-\frac{v}{z}\right)^{\alpha-1} d v
\end{aligned}
$$

où le deuxième membre, fonction de $z$, vérifie l'équation (1.2). Comme $\frac{1}{2} \pi<$ $\theta<\pi$ ou $\pi<\theta<\frac{3}{2} \pi$, l'intégrale définie dans le deuxième membre de (3.5) est certainement convergente (voir [3]). De plus, on voit que le deuxième membre de (3.9) est valable pour $z$ tel que $-\frac{1}{2} \pi<\arg z<\frac{5}{2} \pi$, $\operatorname{car} \theta-\pi<\arg z<\theta+\pi$. On voit aisément que la convergence de (3.9) est localement uniforme sur le domaine $-\frac{1}{2} \pi<$ $\arg z<\frac{5}{2} \pi$, si on regarde la démonstration du lemme 3.4. Ainsi la proposition 3.3 est démontrée.

En unifiant les résultats des propositions 3.1 et 3.3 , on a le Théorème 3.5. Soient $\alpha, \gamma, \theta$ les mêmes constantes que celles qui sont apparues dans le théorème 2.5. Alors, on a le même résultat que celui du théorème 2.5 si on prend la limite $\beta \rightarrow e^{i \theta} \infty$ pour la formule (3.2). 


\section{$\S 4$ Annexe: La représantation intégrale de Pochhammer}

La représantation intégrale suivante pour la série hypergéométrique de Gauss $F(\alpha, \beta, \gamma, z)$ a été obtenue par Pochhammer ([6], [7]).

Lemme 4.1. Soient $\alpha, \beta, \gamma$ les nombres complexes tels que $\alpha \neq 1,2,3, \ldots, \gamma \neq$ $0,-1,-2, \ldots$, et $\gamma-\alpha \neq 1,2,3, \ldots$. Soit $z$ le nombre complexe tel que $1 / z$ soit différent de 0 et de 1 . Alors, on a

$$
\begin{aligned}
F(\alpha, \beta, \gamma, z)= & \frac{\Gamma(\gamma)}{\left(1-e^{2 \pi i(\gamma-\alpha)}\right)\left(1-e^{2 \pi i \alpha}\right) \Gamma(\gamma-\alpha) \Gamma(\alpha)} \\
& \times \int^{(1+, 0+, 1-, 0-)} u^{\alpha-1}(1-u)^{\gamma-\alpha-1}(1-z u)^{-\beta} d u
\end{aligned}
$$

Dans l'intégrale définie du deuxième membre de (4.1), la fonction $u^{\alpha-1}(1-u)^{\gamma-\alpha-1}(1-z u)^{-\beta}$ de la variable $u$ est intégrée le long du cycle de Pochhammer entourant 1 et 0 qui est choisi de façon que l'intérieur avec son bord enclos de ce cycle ne contienne pas le nombre complexe $1 / z$; voici la figure de ce cycle:

Ici, les deux fonctions $u$ et $1-u$ apparaissant dans l'intégrand prennent l'argument 0 en le point $p$ qui est une des extrémités du segment pq, et la fonction $1-z u$ de la variable $z$ prend l'argument 0 en $z=0$.

\section{Bibliographie}

[1] G.E.Andrew, R.Askey, R.Roy, Special Functions, Encyclopedia of Mathematics and its Applications 71, Cambridge Univ. Press, 1999.

[2] E.Goursat, Sur l'équation différentielle linéaire qui admet pour intégrer la série hypergéométrique, Ann. Sci. Ecole Norm. Sup. (2), 10 (1881), S3-S142.

[3] T.Inui, Fonctions spéciales (en japonais), Iwanami, Tokyo, 1962.

[4] E.Kummer, Ueber die hypergeometrische Reihe, J. reine u. angew. Math., 15 (1836), 39-83, 127-172. 
[5] F.W.J.Olver, Asymptotics and Special Functions, A K Peters, Natick, Massachusetts, 1997.

[6] L.Pochhammer, Ueber ein Integral mit doppeltem Umlauf, Math. Ann., 35 (1890), 470-494.

[7] L.Pochhammer, Zur Theorie der Euler'schen Integrale, Math. Ann., 35 (1890), 495-526.

[8] F.G.Tricomi, Fonctions hypergéométriques confluentes, Gauthier-Villars, Paris, 1960.

[9] F.G.Tricomi, A. Erdélyi, The asymptotic expansion of a ratio of Gamma functions, Pacific J. Math., 1 (1951), 133-142. 


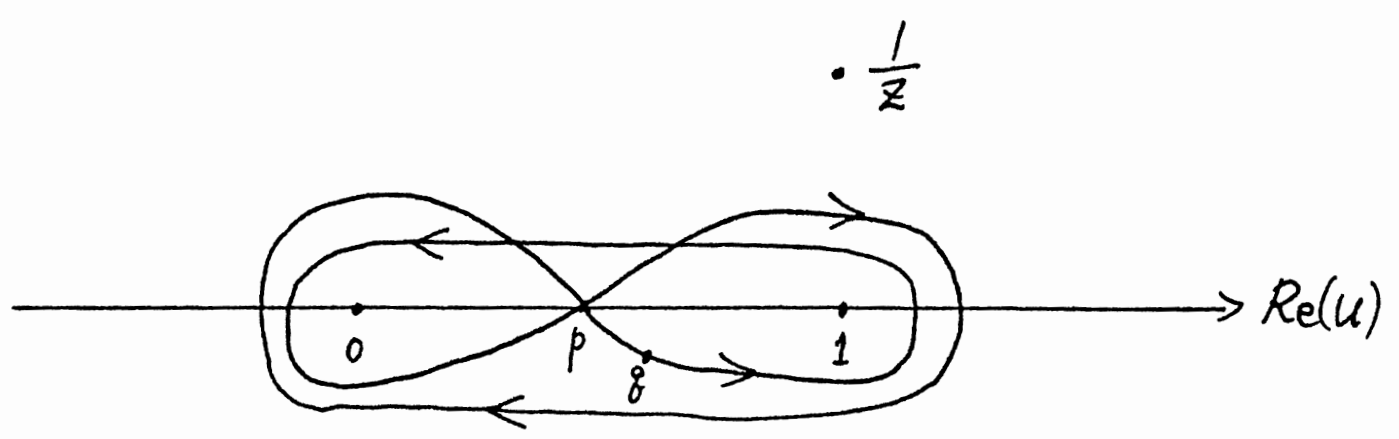

Fig.4.1. 